\title{
Comparison of the results of electrophysiologic testing after short-term and long-term treatment with amiodarone in patients with ventricular tachycardia
}

The results of electrophysiologic testing after short-term and long-term treatment with amiodarone were compared in 71 patients with ventricular tachycardia. Electrophysiologic testing was performed in the baseline state after $11 \pm 3$ days of treatment with 1.2 to $2.4 \mathrm{gm} /$ day of amiodarone, and after $13 \pm 4$ weeks of therapy with a daily amiodarone dose of $400 \mathrm{mg}$. After short-term therapy, $62 \%$ of the patients had an adequate response to amiodarone. In 27 patients who were hemodynamically unstable, ventricular tachycardia was induced and became noninducible or hemodynamically stable after combination therapy with a class I agent. Among 18 patients who did not have inducible ventricular tachycardia after short-term therapy, eight $(44 \%)$ had inducible, hemodynamically unstable ventricular tachycardia after long-term treatment with amiodarone. On the other hand, six of the 27 patients who had hemodynamically unstable ventricular tachycardia after short-term therapy had an adequate response after long-term treatment with amiodarone. Therefore an adequate electrophysiologic response after short-term therapy does not guarantee a similar response after long-term treatment, and an inadequate response after short-term therapy does not always predict a similar response after long-term therapy. (AM HEART J 1991;121:1693.)

Shimon Rosenheck, MD, Joao Sousa, MD, Hugh Calkins, MD, Stephan Schmaltz, MPH, Michael deBuitleir, MD, Alan H. Kadish, MD, and Fred Morady, MD.

Ann Arbor, Mich.

Because amiodarone has a prolonged half-life, several weeks of treatment may be necessary before its maximal electrophysiologic effects become manifest. $^{1-5}$ To hasten the onset of its therapeutic effect, amiodarone has often administered in high dosages for 1 to 2 weeks, after which time electrophysiologic testing has been performed. ${ }^{6-9}$ However, although some previous studies have demonstrated that the results of electrophysiologic testing after 2 to 3 months of treatment with a maintenance does of amiodarone are concordant with the results of testing after short-term treatment, ${ }^{6-8}$ other studies have not confirmed this, ${ }^{10,11}$ and it is unclear whether an

From the Department of Internal Medicine, Division of Cardiology and the Clinical Research Center, University of Michigan Medical Center.

Supported in part by grant 5M01-RR00042-25 from the National Institues of Health, Bethesda, Md.

Received for publication Sept. 18, 1990; accepted Nov. 2, 1990.

Reprint requests: Fred Morady, MD, Division of Cardiology, UH B1F'245, Box 0022, University of Michigan Medical Center, Ann Arbor, MI 48109 0022 .

$4 / 1 / 28512$ electrophysiologic test is clinically necessary after several weeks of therapy with amiodarone. Therefore the purpose of the present study was to compare the results of electrophysiologic testing after 2 weeks and after 2 months of treatment with amiodarone in a large group of patients who had ventricular tachycardia.

\section{METHODS}

Characteristics of patients. The subjects of the study were 71 consecutive patients who were treated with amiodarone for sustained ventricular tachycardia and who underwent electrophysiologic testing after 2 weeks and after 2 to 3 months of treatment with amiodarone. There were 63 men and eight women, with a mean age of $60 \pm 11$ years ( \pm standard deviation). Sixty-six patients had coronary artery disease, two patients had valvular heart disease, and three patients had no evidence of structural heart disease. The mean left ventricular ejection fraction was $0.31 \pm 0.13$. Sixty-six patients had inducible sustained monomorphic ventricular tachycardia during baseline electrophysiologic tests that were performed for evaluation of sustained ventricular tachycardia (47 patients), ventricular fibrilla- 
tion (4 patients), unexplained syncope (7 patients), or nonsustained ventricular tachycardia (8 patients). Five patients did not have a baseline electrophysiologic study in the drug-free state because they were treated with amiodarone after they had had frequent recurrences of ventricular tachycardia that were resistant to other antiarrhythmic agents. Before treatment with amiodarone, the patients underwent unsuccessful electropharmacologic testing with $1.4 \pm 0.7$ class I antiarrhythmic drugs; in addition, a mean of $1.5 \pm 0.8$ other class I antiarrhythmic agents failed to control ventricular tachycardia on a clinical basis.

Amiodarone treatment. The 71 patients were treated orally for $11 \pm 3$ days with 1.2 to $2.4 \mathrm{gm} /$ day amiodarone. The total mean loading dose of amiodarone was $17.3 \pm 6.8$ gm. The mean plasma amiodarone concentration at the time of the first electrophysiologic test was $1.7 \pm 0.7 \mathrm{mg} /$ $\mathrm{ml}$, and the mean desethylamiodarone concentration was $0.9 \pm 0.3 \mathrm{mg} / \mathrm{ml}$. All 71 patients were retested after $13 \pm 4$ weeks of therapy with a daily amiodarone dose of $400 \mathrm{mg}$. After the first electrophysiologic test, 27 patients were treated with a class I antiarrhythmic agent in combination with amiodarone, and in these patients the class I drug was discontinued at least 5 half-lives before the second electrophysiologic test. The mean plasma amiodarone concentration at the time of the second test was $1.4 \pm 0.5 \mathrm{mg} / \mathrm{ml}$ ( $p<0.01$, compared with the concentration at the time of the first test), and the mean desethyl-amiodarone concentration was $1.1 \pm 0.3 \mathrm{mg} / \mathrm{ml}(p<0.001)$.

Electrophysiologic study protocol. Electrophysiologic testing was performed in the fasting, unsedated state (after informed consent was obtained) with the use of two or three quadripolar electrode catheters, which were positioned in the right atrium, the right ventricular apex and outflow tract, and across the tricuspid valve to record the His bundle electrogram. A $5 \mathrm{~F}$ catheter that was introduced into a femoral artery was used to monitor the arterial pressure. Leads $V_{1}, I$, III, and the intracardiac electrograms were recorded on a Siemens Elema Mingograf 7 recorder (Siemens Medical Systems Inc., Iselin, N.J.) at a paper speed of 25 to $100 \mathrm{~mm} / \mathrm{sec}$. Two-millisecond pulses were delivered by a programmable stimulator (Bloom Associates Ltd.), Reading, Pa.). Programmed ventricular stimulation was performed with one to three extrastimuli and a basic drive train duration of 8 beats, a 3- or 4-second intertrain pause, and three basic drive train cycle lengths $(350,400$, 500 , or 600 msec or sinus rhythm). Programmed stimulation was performed first at the right ventricular apex and then at the outflow tract or septum.

Ventricular effective refractory periods were measured by scanning diastole with an extrastimulus in decrements of $10 \mathrm{msec}$. The longest coupling interval that did not result in a ventricular response was considered to be the ventricular effective refractory period. The end point for programmed stimulation was the induction of hemodynamically unstable monomorphic ventricular tachycardia or the completion of the entire protocol. Polymorphic ventricular tachycardia and ventricular fibrillation were not considered end points for testing.
Nonsustained ventricular tachycardia was defined as ventricular tachycardia that was six beats to 30 seconds in duration. Suslained ventricular tachycardia was defined as ventricular tachycardia that was greater than 30 seconds in duration or that required direct-current countershock or pacing to terminate. Hemodynamically stable ventricular tachycardia was defined as ventricular tachycardia that was associated with a mean arterial pressure greater than $65 \mathrm{~mm} \mathrm{Hg}$ with the patient in the supine position. ${ }^{12}$

The criteria that were used to define an adequate response to amiodarone during electrophysiologic testing consisted of the complete suppression of inducible ventricular tachycardia, the induction of only nonsustained ventricular tachycardia that was less than 15 beats in duration, or the induction of sustained ventricular tachycardia that was hemodynamically stable. These criteria were used because previous studies have demonstrated that they are associated with a low risk of arrhythmia-related death in patients who are treated with amiodarone. ${ }^{12}$

Statistical analysis. A mixed model analysis of variance was used to compare the results of electrophysiologic testing in the baseline state and after short-term and long-term therapy with amiodarone. The amiodarone and desethylamiodarone plasma concentrations after short-term and long-term treatment were compared with the use of a paired $t$ test. The results of the electrophysiologic testing after short-term and long-term therapy were compared with Pearson's chi-square test with the Bonferroni's correction for multiple comparisons and Fisher's least significant differences multiple comparisions. A $p$ value of less than 0.05 was considered significant.

\section{RESULTS}

Electrophysiologic effects of amiodarone. The mean sinus cycle length, $\mathrm{AH}$ and $\mathrm{HV}$ intervals, QRS duration, and QT interval increased significantly from baseline values after short-term therapy with amiodarone and remained unchanged after long-term therapy (Table I). At a basic drive cycle length of 600 msec, the ventricular effective refractory period increased from the baseline value by a mean of $17 \% \pm 12 \%(p<0.0001)$ after short-term treatment with amiodarone and by $14 \% \pm 11 \%(p<0.0001)$ after long-term treatment. There was no significant change in the ventricular effective refractory period between the short-term and long-term tests. The results were similar at a basic drive train cycle length of $400 \mathrm{msec}$ (Table I).

Baseline ventricular tachycardia characteristics. Sixty-six patients underwent baseline electrophysiologic tests in the absence of antiarrhythmic drug therapy. The mean cycle length of ventricular tachycardia that was induced in the baseline test was $285 \pm 56 \mathrm{msec}$, and the mean arterial pressure during the period of induced ventricular tachycardia was $47 \pm 17 \mathrm{~mm} \mathrm{Hg}$. In 10 patients, the mean arterial pressure during ventricular tachycardia ranged be- 
tween 65 to $80 \mathrm{~mm} \mathrm{Hg}$ (mean $76 \pm 8 \mathrm{~mm} \mathrm{Hg}$ ), and in these patients the mean ventricular tachycardia cycle length was $336 \pm 50 \mathrm{msec}$. In the other 56 patients, the mean ventricular tachycardia cycle length was $274 \pm 49 \mathrm{msec}$, and the mean arterial pressure ranged between 20 and $63 \mathrm{~mm} \mathrm{Hg}$ (mean $41 \pm 12 \mathrm{~mm} \mathrm{Hg}$ ).

Short-term effect of amiodarone on the induction of ventricular tachycardia. Among the 71 patients who underwent electrophysiologic tests after $11 \pm 3$ days of amiodarone treatment, 44 had an adequate response to amiodarone; no ventricular tachycardia could be induced in 18 patients, only nonsustained ventricular tachycardia was induced in eight patients, and hemodynamically stable ventricular tachycardia was induced in 18 patients. In the remaining 27 patients, hemodynamically unstable ventricular tachycardia, which had a mean cycle length of $346 \pm 58 \mathrm{msec}$ and which was associated with a mean arterial pressure of $50 \pm 8 \mathrm{~mm} \mathrm{Hg}$, was induced. In these patients, a class I antiarrhythmic agent was combined with amiodarone to obtain a satisfactory effect on the inducibility of ventricular tachycardia. Amiodarone was used with mexiletine in eight patients, with quinidine in nine patients, with encainide in seven patients, with flecainide in two patients, and with tocainide in one patient. In one patient ventricular tachycardia was noninducible and in another only nonsustained ventricular tachycardia could be induced. In the remaining 25 patients, a slower and hemodynamically stable ventricular tachycardia was induced. The mean ventricular tachycardia cycle length in these 26 patients increased from $347 \pm 57 \mathrm{msec}$ to $411 \pm 71 \mathrm{msec}(p<0.01)$, and the mean arterial pressure increased from $50 \pm 8 \mathrm{~mm}$ $\mathrm{Hg}$ to $75 \pm 14 \mathrm{~mm} \mathrm{Hg}(p<0.001)$ after addition of the class I antiarrhythmic agent.

Long-term effect of amiodarone on induction of ventricular tachycardia. All patients underwent another electrophysiologic test, after $13 \pm 4$ weeks of therapy with amiodarone, to evaluate the efficacy of longterm treatment with amiodarone in preventing the induction of hemodynamically unstable ventricular tachycardia. In 34 patients (45\%), amiodarone was effective in suppressing the induction of hemodynamically unstable ventricular tachycardia, and in 39 patients $(55 \%)$ it was not. The mean cycle length of the induced ventricular tachycardia in these 39 patients was $332 \pm 56 \mathrm{msec}$, and the mean arterial pressure during the ventricular tachycardia was $42 \pm 11 \mathrm{~mm} \mathrm{Hg}$. Ten of the 39 patients underwent implantation of an automatic cardioverter/defibrillator; two other patients did not give consent for a defibrillator implantation and continued on combi-
Table I. The effect of amiodarone on sinus cycle length; nodal, infranodal, and intraventricular conduction; and repolarization and ventricular effective refractory periods

\begin{tabular}{lccc}
\hline & Baseline & $\begin{array}{c}\text { Short-term } \\
\text { amino }\end{array}$ & $\begin{array}{c}\text { Long-term } \\
\text { amino }\end{array}$ \\
\hline SCL (msec) & $853 \pm 176$ & $939 \pm 184^{*}$ & $955 \pm 190^{*}$ \\
AH (msec) & $108 \pm 35$ & $122 \pm 41^{*}$ & $119 \pm 45^{*}$ \\
HV (msec) & $53 \pm 14$ & $61 \pm 17 \dagger$ & $64 \pm 23 \dagger$ \\
QRS (msec) & $113 \pm 27$ & $128 \pm 30^{*}$ & $129 \pm 31^{*}$ \\
QT (msec) & $391 \pm 27$ & $460 \pm 70^{*}$ & $456 \pm 50^{*}$ \\
VERP at 600 & $254 \pm 20$ & $293 \pm 27^{*}$ & $291 \pm 23^{*}$ \\
$\quad$ msec & & & \\
VERP at 400 & $238 \pm 18$ & $272 \pm 26^{*}$ & $272 \pm 20^{*}$ \\
$\quad$ msec & & & \\
\hline
\end{tabular}

There were no significant differences between short-term and long-term therapy with amiodarone.

Short-term amio, Electrophysiologic study after $11 \pm 3$ days a high-dose amiodarone treatment; Long-term amio, electrophysiologic study after $12+3$ weeks of amiodarone treatment; $\mathrm{SCl}$, sinus rycle length; $\mathrm{AH}, \mathrm{AH}$ Interval duration; HV, HV interval duration; VERP, ventricular effective refractory period.

${ }^{*} p<0.0001$ compared with baseline.

$t p<0.05$ compared with baseline.

nation therapy with quinidine or encainide. Twentyseven patients had long-term therapy with a combination of amiodarone and a class I antiarrhythmic agent.

Comparison of the results of the testing after shortterm and after long-term therapy. Among 44 patients who had favorable responses during electrophysiologic testing after short-term therapy with amiodarone, $18(41 \%)$ no longer had favorable responses after long-term therapy and had inducible sustained, hemodynamically unstable ventricular tachycardia. On the other hand, among the 27 patients who had inadequate responses after short-term therapy, 6 $(22 \%)$ had favorable responses after long-term therapy and no longer had inducible hemodynamically unstable ventricular tachycardia. These results are summarized in Table II.

Of the 18 patients who did not have inducible ventricular tachycardia after short-term therapy, ventricular tachycardia remained noninducible after long-term therapy in only six. Eight of the 18 patients (44\%) had inducible ventricular tachycardia, which was hemodynamically unstable after long-term therapy.

Overall, in $34 \%$ of the 71 patients in this study, the results of programmed ventricular stimulation after short-term therapy with amiodarone did not accurately predict the results after long-term therapy. None of these patients experienced hemodynamically unstable ventricular tachycardia or sudden death during the interval between the two tests. 
Table Il. Comparison of the response to programmed ventricular stimulation after short-term and long-term therapy with amiodarone in 71 patients

\begin{tabular}{lrrccc}
\hline \multicolumn{1}{c}{ Short-term therapy } & \multicolumn{4}{c}{ Long-term therapy* } \\
\hline No VT & 18 & 6 & 2 & 2 & 8 \\
Non-sust VT & 8 & 3 & 1 & 0 & 4 \\
Stable VI & 18 & 2 & 1 & 9 & 6 \\
Unstable VT & $\frac{27}{71}$ & $\frac{2}{13}$ & $\frac{1}{5}$ & $\frac{3}{14}$ & $\frac{21}{39}$ \\
$\quad$ TOTALS & & &
\end{tabular}

VT, Ventricular tachycardia. Non-sust, nonsustained. ${ }^{*} p<0.001$ compared with short-term therapy.

Table III. The effect of amiodarone on the cycle length of the induced sustained and nonsustained ventricular tachycardia and on the blood pressure during the tachycardia

\begin{tabular}{lccc}
\hline \multicolumn{1}{c}{ Condition } & Patients & $\begin{array}{c}\text { VTCL } \\
\text { (msec) }\end{array}$ & $\begin{array}{c}B P \\
(m m ~ H g)\end{array}$ \\
\hline Baseline & 63 & $285 \pm 56$ & $47 \pm 17$ \\
Short-Term Amio & 51 & $365 \pm 56^{*}$ & $66 \pm 20^{*}$ \\
Long-Term Amio & 56 & $361 \pm 70^{*}$ & $56 \pm 22 \dagger$ \\
\hline
\end{tabular}

VTCL, Mean cycle length of the ventricular tachycardia; BP, blood pressure during the tachycardia; other abbreviations as in Table I.

${ }^{*} p<0.0001$ compared with baseline.

$\dagger p<0.01$ compared with baseline and $p<0.001$ compared with Short-term amio.

However, three patients had documented episodes of hemodynamically stable ventricular tachycardia during this interval.

Among 45 patients with documented ventricular tachycardia, the clinical ventricular tachycardia was reproduced during the baseline electrophysiologic tests in $64 \%$. After short-term therapy, ventricular tachycardia could be induced in $75 \%$ of patients, and in $47 \%$ of them, the tachycardia had the same morphology as the ventricular tachycardia that was induced in the baseline state. After long-term therapy with amiodarone, ventricular tachycardia could be induced in $82 \%$ of the patients, and in $60 \%$ of them the tachycardia had the same morphology as the ventricular tachycardia that was induced in the baseline state.

Among the patients with inducible ventricular tachycardia after short-term therapy with amiodarone, the ventricular tachycardia cycle length increased by $30 \% \pm 30 \%$ compared with the baseline state ( $p<0.0001$ ), and among the patients with inducible ventricular tachycardia after long-term therapy with amiodarone, the tachycardia cycle length increased by $29 \% \pm 23 \%$ compared with the baseline state $(p<0.0001)$. There was no significant difference in the tachycardia cycle length between short-term and long-term amiodarone therapy (Table III).

The mean blood pressure during the tachycardia increased by $53 \% \pm 73 \%$ between the baseline state and the early follow-up study $(p<0.0001)$, but by only $27 \% \pm 73 \%$ between the baseline state and the late follow-up study $(p<0.01)$. The decrease of $11 \% \pm 37 \%$ between the early and the late studies was also significant $(p<0.001)$. There was a significant linear correlation between the ventricular tachycardia cycle length and the mean blood pressure in all three studies $(p<0.01)$.

\section{DISCUSSION}

Main findings. This study demonstrates that the results of electrophysiologic testing after short-term and long-term treatment with amiodarone are discordant in $34 \%$ of patients who are treated for sustained ventricular tachycardia. In $22 \%$ of patients in whom hemodynamically unstable, sustained monomorphic ventricular tachycardia was inducible after approximately 10 days of treatment, sustained ventricular tachycardia was either no longer inducible or was inducible but slower and hemodynamically state 2 months later. On the other hand, among $41 \%$ of patients who demonstrated an adequate response to amiodarone after short-term therapy, the response to amiodarone during electrophysiologic testing $13 \pm 4$ weeks later was no longer adequate, and hemodynamically unstable ventricular tachycardia was inducible. These findings demonstrate that the results of electrophysiologic testing after a high loading dose regimen may not accurately reflect the results that are found after several weeks of treatment with a maintenance dose of $400 \mathrm{mg} / \mathrm{day}$ and indicate the importance of reevaluating patients after 3 months of therapy, even when the clinical course during the first 2 or 3 months of therapy has been benign. In contrast to the variable effect of amiodarone on the inducibility and hemodynamic stability of ventricular tachycardia after short-term and long-term treatment, its effect on measures of conduction and ventricular refractoriness were the same after 10 days and after 3 months of therapy.

Prognostic implications of the results of electrophysiologic testing after short-term amiodarone therapy. Of note is that none of the patients in this study experienced hemodynamically unstable ventricular tachycardia in the interval between the electrophysiologic tests that were performed after short-term and after long-term therapy with amiodarone. Therefore although an acceptable response during electrophysiologic testing after short-term therapy with amio- 
darone does not guarantee that the response will still be acceptable 3 months later, the absence of inducible ventricular tachycardia that is hemodynamically unstable after short-term treatment suggest that the patient may be safely discharged from the hospital to await a repeat electrophysiologic test 3 months later.

Electrophysiologic effects of amiodarone. Amiodarone prolongs the sinus cycle length and slows conduction throughout the heart. ${ }^{1-3,7-9,13-16}$ With a loading dose of amiodarone for 1 to 2 weeks and then a maintenance dose for 1 to 3 months, the prolongation in sinus cycle length and the slowing of conduction has been found to be maximal after 1 to 2 weeks of therapy. ${ }^{6-8,14,17,18}$ The present study confirms this observation.

In early experimental studies, when treatment with amiodarone was started at a low dosage, prolongation of the ventricular action potential was gradual in onset and maximal only after 6 weeks of treatment. ${ }^{1,3,5}$ In contrast, in studies in which a loading dose of amiodarone preceded treatment with a maintenance dose, significant electrophysiologic effects were present and often maximal after 1 to 2 weeks of treatment. ${ }^{6-8,14,18,19}$ In the present study, the mean total amiodarone loading dose was $17.3 \pm 6.8 \mathrm{gm}$, which is markedly higher than the loading doses that were used in previous studies. With this high loading dose of amiodarone, the electrophysiologic effects of amiodarone were maximal after approximately 10 days of treatment. Therefore although the effects of amiodarone on the results of programmed ventricular stimulation after 3 months of treatment cannot be accurately predicted after 10 days of treatment, the effects on the sinus cycle length, on parameters of conduction, and on the ventricular effective refractory period are already maximal after short-term treatment when a large loading dose is used. The discrepancy between the effects of amiodarone on measures of conduction and refractoriness and the antiarrhythmic effect of amiodarone indicates that global measures of conduction and right ventricular refractoriness may not accurately reflect changes in the electrophysiologic properties of the recntry circuit.

Effects of amiodarone on ventricular tachycardia and comparison with previous studies. The timing of the maximal antiarrhythmic effect of amiodarone has been the subject of several recent studies. ${ }^{6-8,10}, 11,19$ These studies demonstrate that the response to amiodarone during electrophysiologic testing may be better after long-term therapy than after short-term therapy. However, none of these studies included patients who had acceptable responses to amiodarone after short-term therapy. Previous studies have had a population size of 20 to 34 patients and have included patients who had inducible ventricular fibrillation in the baseline electrophysiologic test. ${ }^{6,7,10}$ Only the most recent studies included hemodynamic criteria for assessment of the antiarrhythmic effect of amiodarone. ${ }^{6,17}$ In contrast to previous studies, the present study included 71 patients, each of whom had inducible, sustained, monomorphic ventricular tachycardia in the baseline state and each of whom underwent electrophysiologic testing after long-term treatment, even when a favorable response had occurred after short-term treatment.

Limitations. A limitation of this study is that it does not provide direct evidence that the results of electrophysiologic testing after 2 to 3 months of amiodarone therapy are clinically significant. When hemodynamically unstable ventricular tachycardia was inducible, patients either received combination therapy with a second antiarrhythmic drug or underwent implantation of an automatic defibrilator, in which case amiodarone therapy was discontinued or tapered in dosage. Therefore the natural history of patients who demonstrated adequate responses to shortterm therapy but inadequate responses to long-term therapy with amiodarone was not established in this study.

Clinical implications. The results of this study have important clinical implications for the management of patients with life-threatening ventricular tachycardia who are treated with amiodarone. In patients in whom sustained and hemodynamically unstable ventricular tachycardia is inducible after 1 to 2 weeks of treatment with a loading dose of amiodarone, combination therapy with a class I antiarrhythmic drug may result in slowing of the ventricular tachycardia that is sufficient to result in hemodynamic stability during ventricular tachycardia. If this is the case, the patient may be safely discharged from the hospital with treatment that consists of the combination of the class I drug and a maintenance dose of amiodarone. Repeat testing 2 to 3 months later, after discontinuation of treatment with the class I drug, may demonstrate that amiodarone by itself is sufficient to suppress the induction of ventricular tachycardia or to result in the induction of sustained ventricular tachycardia that is hemodynamically stable. These patients may then be safely treated on a longterm basis with amiodarone by itself, whereas the other patients may require continued treatment with a second drug or may be appropriate candidates for implantation of an automatic implantable cardioverter-defibrillator. Some patients who demonstrate an adequate response to amiodarone during electro- 
physiologic testing after 2 to 3 months of therapy might otherwise have been thought to be appropriate candidates for implantation of an autonomic cardioverter-defibrillator, if the decision to implant the defibrillator were based on the results of electrophysiologic testing that was performed after short-term therapy.

The results of this study also indicate the importance of a follow-up electrophysiologic test after 2 to 3 months of therapy with amiodarone in patients who demonstrate adequate short-term responses to amiodarone. More than one third of patients who initially respond adequately to amiodarone may be found to have inducible sustained ventricular tachycardia that is hemodynamically unstable 2 to 3 months later and may therefore be appropriate candidates for therapy with a second antiarrhythmic drug or for implantation of an autonomic implantable cardioverter-defibrillator.

It should be stressed that only patients in whom ventricular tachycardia was noninducible or inducible but hemodynamically stable after short-term therapy with amiodarone by itself or with a second drug were included in this study. Our results do not imply that the final decision to implant a defibrillator in a patient who has been treated with amiodarone for life-threatening ventricular tachycardia can always be safely postponed for 2 to 3 months after an initial electrophysiologic test that demonstrates an inadequate response to amiodarone. Patients in whom sustained and hemodynamically unstable ventricular tachycardia remains inducible after shortterm therapy with amiodarone may not tolerate or respond to combination therapy with a second antiarrhythmic agent and may be appropriate immediate candidates for nonpharmacologic therapy, such as implantation of an autonomic defibrillator.

The authors thank Marion Maguire for her excellent assistance in the preparation of the manuscript.

\section{REFERENCES}

1. Singh BN, Venkatesh N, Nademanee K, Josephson MA, Kannan R. The historical development, cellular electrophysiology and pharmacology of amiodarone. Prog Cardiovasc Dis 1989; 31:249-80.

2. Zipes PD, Prystowsky EN, Heger JJ. Amiodarone: electrophysiologic action, pharmacokinetics and clinical effects. J Am Coll Cardiol 1984;4:1059-71.

3. Ikeda N, Nademanee K, Kannan R, Singh BN. Electrophysiologic effects of amiodarone: experimental and clinical observations relative to serum and tissue drug concentrations. AM HEART J 1984;108:890-8.

4. Nademanee K, Hendrickson JA, Cannom DS, Goldreyer BN,
Singh BN. Control of refractory life-threatening ventricular tachyarrhythmias by amiodarone. AM HEART J 1981;101: 75968.

5. Singh BN, Vaughan Williams EM. The effect of amiodarone, a new antianginal drug, on cardiac muscle. Br J Pharmacol 1970;39:657-67.

6. Mitchell LB, Wyse G, Gillis AM, Duff HJ. Electropharmacol ogy of amiodarone therapy initiation: time course of onset of electrophysiologic and antiarrhythmic effects. Circulation 1989;80:34-42.

7. Kadish AH, Marchlinski FE, Josephson ME, Buxton AE. Amiodarone: correlation of early and late electrophysiologic studies with outcome. AM HEART J 1986;112:1134-40.

8. Kennedy EE, Rosenfeld LE, McPherson CA, Batsford WP. Evaluation by serial electrophysiologic studies of an abbreviated oral loading regimen of amiodarone. Am J Cardiol 1985; 56:867-71.

9. Heger JJ, Prystowsky EN, Jackman WM, Naccarelli GV, Warfel KA, Rinkenberger RL, Zipes DP. Amiodarone: clinical efficacy and electrophysiology during long-term therapy for recurrent ventricular tachycardia or ventricular fibrillation. $\mathrm{N}$ Engl J Med 1981;305:539-45.

10. Greenspon AJ, Volosin KJ, Greenberg RM, Jefferies L, Rot mensch HH. Amiodarone therapy: role of early and late electrophysiologic studies. J Am Coll Cardiol 1988;11:117-23.

11. Krafchek J, Lin HT, Beckman KJ, Nielsen AP, Magro SH, Hargis J, Wyndham CRC. Cumulative effect of amiodarone on inducibility of ventricular tachycardia: implications for electrophysiologycal testing. PACE 1988;11:434-44.

12. Waller TJ, Kay HR, Spielman SR, Kutalek SP, Greenspan AM, Horowitz L.N. Reduction in sudden death and total mortality by antiarrhythmic therapy evaluated by electrophysiologic drug testing: criteria of efficacy in patients with sustained ventricular tachyarrhythmia. J Am Coll Cardiol 1987;10: 83-9.

13. Veltri PE, Reid PR, Platia EV, Griffith LSC. Results of late programmed electrical stimulation and long-term electrophysiological effects of amiodarone therapy in patients with refractory ventricular tachycardia. Am o Cardiol 1985;55: 375-9.

14. Horiwitz LA, Greenspan AM, Spielman SR, Webb C, Morganroth J, Rotmensch H, Sokoloff NM, Rae AP, Segal BL, Kay HR. Usefulness of electrophysiologic testing in evaluation of amiodarone therapy for sustained ventricular tachyarrhythmias associated with coronary heart disease. Am J Cardiol 1985;55:367-71.

15. Waxman HL, Groh WC, Marchlinski FE, Buxton AE, Sadowski LM, Horowitz LN, Josephson ME, Kastor JA. Amiodarone for control of sustained ventricular tachyarrhythmia: clinical and electrophysiologic effect in 51 patients. Am J Cardiol 1982;50:1066-74.

16. Nademanee K, Hendrickson J, Kannan R, Singh BN. Antiarrhythmic efficacy and electrophysiologic action of amiodarone in patients with life-threatening ventricular arrhythmias: potent suppression of spontaneously occurring tachyarrythmias versus inconsistent abolition of induced ventricular tachycardia. AM HEART J 1982;103:950-9.

17. Kadish AH, Buxton AE, Waxman HL, Flores B, Josephson ME, Marchlinski FE. Usefulness of electrophysiologic study to determine the clinical tolerance of arrhythmia recurrences during amiodarone therapy. J Am Coll Cardiol 1987;10:90-6.

18. DiCarlo LA Jr, Morady F, de Buitleir M, Baerman JM, Schuring L, Annesley $T$. Effect of chronic amiodarone therapy on ventricular tachycardia induced by programmed ventricular stimulation. AM HEART J 1987;113:57-64.

19. Greenberg ML, Lerman BB, Haines DE, Baron JA, DiMarco JP. Stability of electrophysiological parameters after acute amiodarone loading: implications for patient management. PACE 1989;12:1038-43. 